\title{
Early postnatal exposure to intermittent hypoxia in rodents is proinflammatory, impairs white matter integrity, and alters brain metabolism
}

\author{
Robert A. Darnall ${ }^{1,2}$, Xi Chen $^{3}$, Krishnamurthy V. Nemani ${ }^{3}$, Chrystelle M. Sirieix ${ }^{1}$, Barjor Gimi ${ }^{3}$, Susan Knoblach ${ }^{4}$, \\ Betty L. McEntire ${ }^{5}$ and Carl E. Hunt ${ }^{4,6}$
}

BACKGROUND: Preterm infants are frequently exposed to intermittent hypoxia $(\mathrm{IH})$ associated with apnea and periodic breathing that may result in inflammation and brain injury that later manifests as cognitive and executive function deficits. We used a rodent model to determine whether early postnatal exposure to $\mathbb{H}$ would result in inflammation and brain injury.

METHODS: Rat pups were exposed to $\mathbb{H}$ from P2 to P12. Control animals were exposed to room air. Cytokines were analyzed in plasma and brain tissue at P13 and P18. At P20$P 22$, diffusion tensor imaging (DTI) and magnetic resonance spectroscopy (MRS) were performed.

RESULTS: Pups exposed to $\mathrm{H}$ had increased plasma Gro/ CXCL1 and cerebellar IFN- $\gamma$ and IL-1 $\beta$ at P13, and brainstem enolase at P18. DTI showed a decrease in FA and AD in the corpus callosum (CC) and cingulate gyrus, and an increase in $\mathrm{RD}$ in the CC. MRS revealed decreases in NAA/Cho, $\mathrm{Cr}$, Tau/Cr, and $\mathrm{Gly} / \mathrm{Cr}$; increases in TCho and GPC in the brainstem; and decreases in NAA/Cho in the hippocampus.

CONCLUSIONS: We conclude that early postnatal exposure to $\mathbb{H}$, similar in magnitude to that experienced in human preterm infants, is associated with evidence for proinflammatory changes, decreases in white matter integrity, and metabolic changes consistent with hypoxia.

$\mathbf{P}$ remature infants have repetitive bouts of intermittent hypoxia $(\mathrm{IH})$ associated with immature respiratory control, including apnea and periodic breathing, which continue until about 42-43 weeks postmenstrual age (PMA) $(1,2)$. With maturation, much of the IH is due to periodic breathing (3), and is often not accompanied by large heart rate decelerations, in contrast to IH during longer apneas where bradycardia is common. Repetitive cycles of re-oxygenation following each $\mathrm{IH}$ episode cause oxidative stress, free-radical production, and the release of proinflammatory cytokines (4).
In extremely premature infants, elevated inflammatory biomarkers in the first two postnatal weeks are associated with bronchopulmonary dysplasia, developmental delays, and cerebral palsy $(5,6)$. The reasons for the elevations in inflammatory biomarkers remain unclear and the relationship between $\mathrm{IH}$, elevations in inflammatory biomarkers, and neurodevelopmental impairment has not been studied. IH associated with obstructive sleep apnea (OSA) in children and adults, however, is associated with increased levels of inflammatory biomarkers (7), magnetic resonance imaging (MRI) abnormalities, and significant cognitive and executive function deficits (7). It is unknown, however, whether IH persisting until $42-43$ weeks PMA in preterm infants is associated with brain inflammation, MRI abnormalities, and/ or significant cognitive and executive function deficits.

The long-term physiological effects of IH during the neonatal period have been studied extensively in rodent models (8-10), but few studies have focused on brain development and/or injury, or on brain inflammation. However, two studies in mice have indicated that early postnatal exposure to relatively mild IH shows biochemical and electron microscopy evidence for a decrease in myelination possibly due to impaired myelinogenesis, impaired axonal maturation, short- and long-term neurofunctional deficits, and electrophysiological changes that may form the basis for more long-term neurobehavioral sequelae $(11,12)$. An additional study in rats examined the effects of postnatal hyperoxia combined with mild $\mathrm{IH}$ on retinopathy of prematurity and reported transient MRI changes in radial (RD) and axial (AD) diffusivity, and fractional anisotropy (FA) in certain white matter regions that did not persist beyond P14 (ref. 13) These findings suggest that there may be adverse effects of IH on white matter development. Inflammatory markers were not investigated in these studies, and there is little or no information specifically implicating a causal relationship between $\mathrm{IH}$ and changes in inflammatory markers during early development. The current study was 

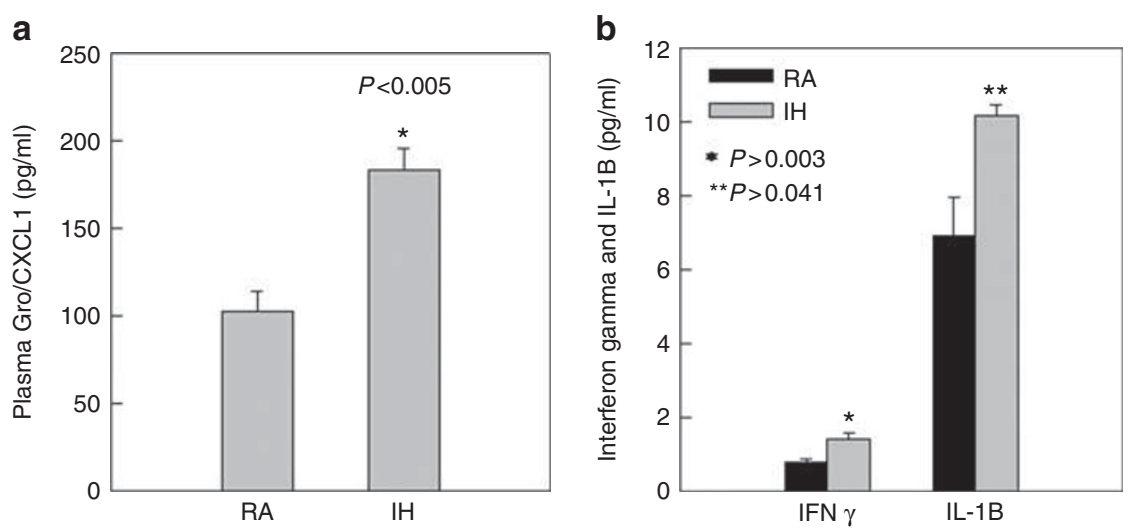

Figure 1. Inflammatory biomarker results at $\mathrm{P} 13$ in rat pups exposed to $\mathrm{IH}$ at $\mathrm{P} 2-\mathrm{P} 12$, compared with normoxic controls. (a) Plasma concentrations of GRO/CXCL1 (pg/ml). (b) Cerebellar concentrations of cerebellar IFN- $\gamma$ and IL-1 $\beta$ (pg/ml).

designed to determine in rodent pups whether exposure to a level of $\mathrm{IH}$ analogous in severity and timing to that occurring in human infants born prematurely causes changes in plasma and/or brain inflammatory markers, brain injury markers, and/or changes in MRI findings. We hypothesized that postnatal exposure to IH in rodent pups would result in systemic and brain parenchymal inflammation and/or brain injury, and we obtained MRI evidence for decreases in white matter integrity and persistent metabolic changes consistent with exposure to hypoxia.

\section{RESULTS}

\section{Inflammatory Cytokines}

Gro/CXCL1, a chemokine that primarily acts on neutrophils and in the CNS inhibits migration of oligodendrocyte progenitor cells, was elevated in the plasma of IH pups at P13 compared with RA pups $(P<0.005$; Figure 1a). In addition, Gro/CXCL1 was detectable in only $2 / 10$ samples from the cerebellum in the RA group, whereas all samples from the cerebellum in the IH group had detectable values greater than the two samples in the RA group. Not all brain regions tested had elevated cytokine levels. However, in $\mathrm{IH}-$ exposed pups, IFN- $\gamma$ and IL- $1 \beta$ were increased in the cerebellum compared with RA pups at P13 $(P<0.003$ and $P=0.041$, respectively, Figure $1 \mathbf{b}$ ). There were no significant increases in TNF-a, IFN- $\gamma$, or IL-10 (data not shown), and other cytokines were below the limit of detection of the assay. Moreover, there were no significant increases in cytokines in tested brain regions, or in plasma, at P18. These data indicate the presence of acute brain parenchymal and systemic inflammation after exposure to $\mathrm{IH}$, which was absent by P18.

\section{Brain Injury}

There were no significant IH-related changes in NSE associated with brain injury at P13. However, at P18, NSE was significantly higher in the medullas of IH compared with RA pups $(1319.7 \pm 124 \mu \mathrm{g}$ vs $802.3 \pm 124 \mu \mathrm{g}, P=0.034)$. Collectively, the inflammatory cytokine and brain injury biomarker data support the presence of IH-induced acute systemic and cerebellar inflammation that did not persist and evidence for delayed medullary injury present at P18.

\section{DTI}

FA maps for an IH and an RA pup indicating a decrease in density in the corpus callosum and cingulate are shown in Figure $2 \mathbf{b}$. Figure 3 shows values for $\mathrm{RD}, \mathrm{AD}, \mathrm{MD}$, and $\mathrm{FA}$ for all of the animals for all of the chosen regions of interest. $\mathrm{FA}$ and $\mathrm{AD}$ were significantly decreased in the corpus callosum (CC) and cingulate gyrus (CG; at least $P<0.008$ ), whereas $\mathrm{RD}$ increased significantly in the CC $(P=0.047)$. Overall, our findings indicate decreases in $\mathrm{FA}$ and $\mathrm{AD}$ and increases in $\mathrm{RD}$ in major central white matter regions, consistent with axonal injury and de- and/or dys-myelination.

\section{MRS}

Although 20 metabolites were quantified, we focused on markers for cellular bioenergetics (creatine, Cr), neuronal integrity ( $N$-acetyl-aspartate, NAA), membrane turnover (choline, Cho), neurotransmission (glycine, Gly), and neuroprotection (taurine, Tau). A typical ${ }^{1} \mathrm{H}$ spectrum from the hippocampus in a pup exposed to IH is shown in Figure 4. The inset shows the area including the hippocampus, which was included in the analysis. In the brainstem, the concentration of $\mathrm{Cr}$ decreased significantly in pups exposed to IH compared with RA controls $(P=0.031)$. There was also an increase in total $\mathrm{Cr}$ ( $\mathrm{TCr}$ ) content (Cr+phospho (p) $\mathrm{Cr}$ ) $(P=0.028)$ consistent with their role in maintaining ATP levels required for the protection of brain tissue from hypoxic insult (14).

NAA/Cho significantly decreased in both the brainstem $(P=0.042)$ and the hippocampus $(P=0.037)$. There were also related but not statistically significant decreases in the ratios of NAA containing metabolites to $\mathrm{Cr}$ in the brainstem and hippocampus. These data strongly suggest that exposure to $\mathrm{IH}$ was associated with decreases in neuronal integrity. There was also a significant increase in total Cho (TCho) (Cho+pCho) 


\section{Articles | Darnall et al.}
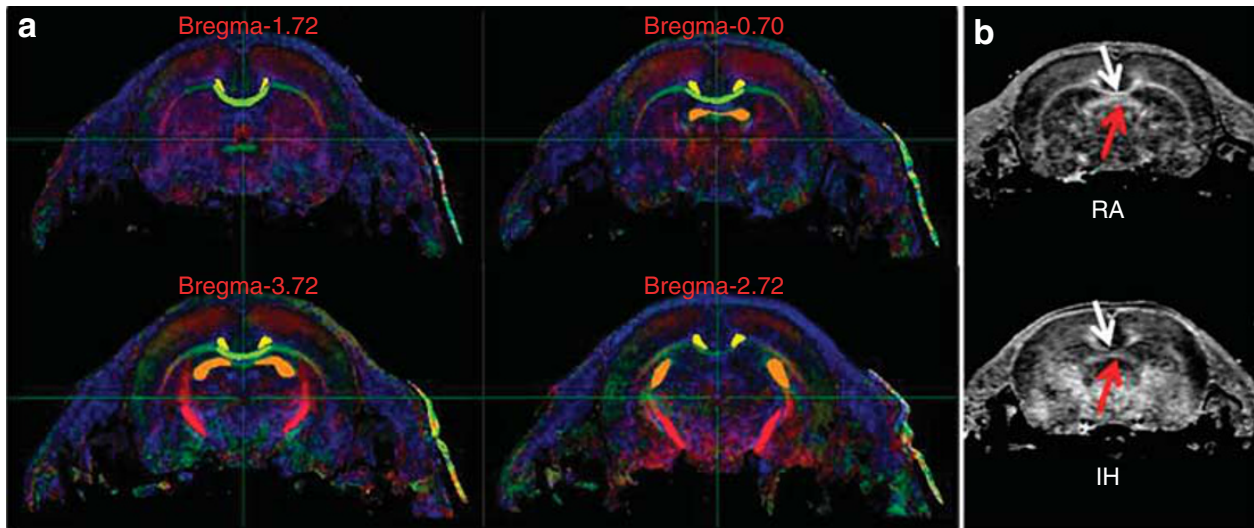

Figure 2. (a) Regions of Interest (ROI) for DTI studies shown in several coronal planes. Corpus Callosum (CC) (green); Cingulate Gyrus (CG) (orange); Fimbria (F) (yellow); Internal Capsule (IC) (red). (b) Example FA maps for an IH and RA control pup. Note in particular the decrease in density of the CC (corpus callosum) (white arrows) and CG (cingulate gyrus) (red arrows).
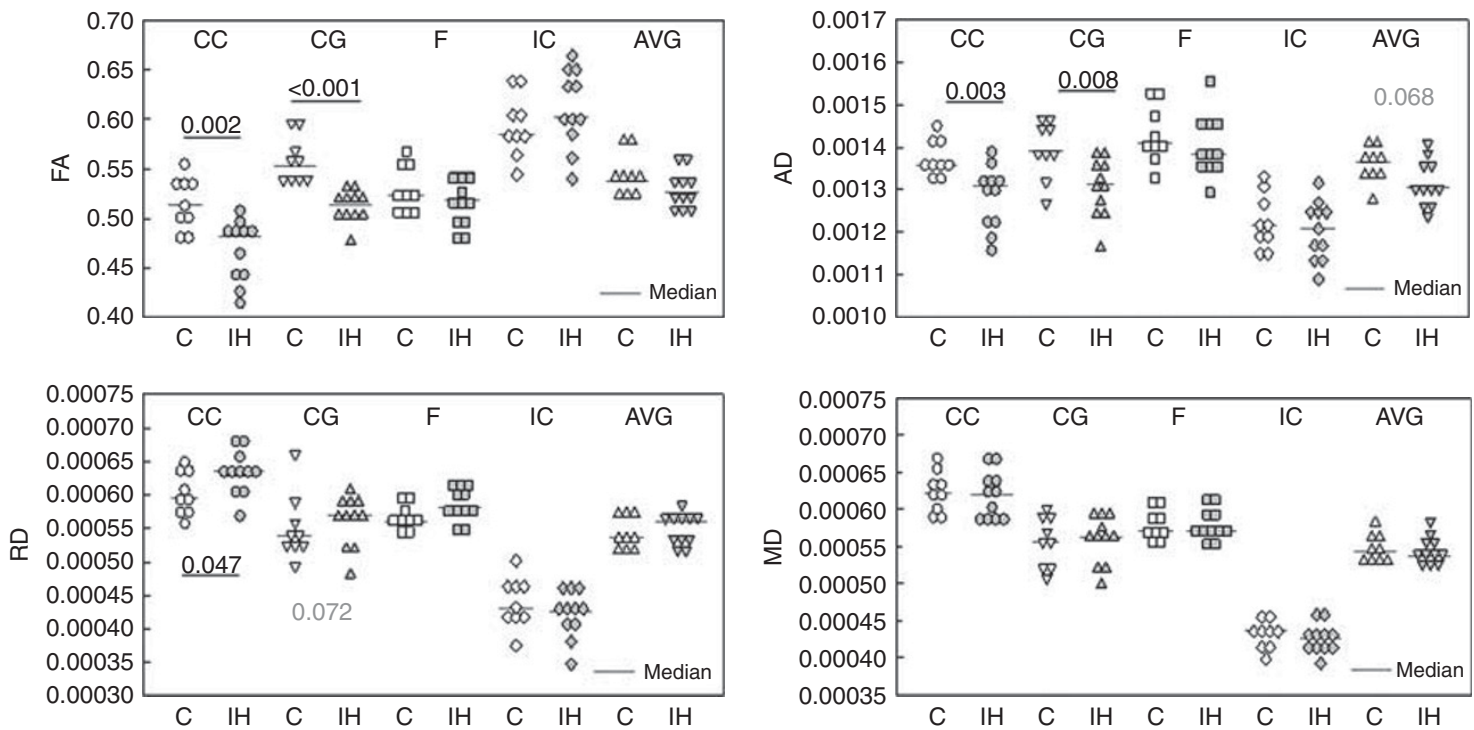

Figure 3. Changes in $F A, A D, R D$, and $M D$ for the regions of interest (CC, corpus callosum; CG, cingulate gyrus; $F$, fimbria; IC, internal capsule; $A V G$, average across all regions). Gray symbols represent pups exposed to $\mathrm{IH}$. Open symbols are room-air-exposed control pups. Blue lines indicate the medians. Significant values are shown in bold underline $(P<0.05)$. Nonsignificant changes with $P \leq 0.08$ are shown in gray.

$(P<0.001)$ and a consistent but nonsignificant increase in pCho in the brainstem accompanied by the suggestion of a decrease in Glycerophosphocholine (GPC) in the hippocampus in the IH pups. Together, these changes suggest that exposure to $\mathrm{IH}$ was associated with an increase in membrane turnover.

Importantly, Tau is thought to play a neuroprotective role, and a decrease of Tau/Cr $(P=0.027)$ in the brainstem after exposure to IH may indicate an increased vulnerability to hypoxic injury. In addition, there was an increase in the concentration of glycine (Gly) $(P=0.031)$ in the hippocampus and a decrease in $\mathrm{Gly} / \mathrm{Cr}$ in the brainstem $(P=0.037)$, suggesting an effect of $\mathrm{IH}$ on neurotransmission. Results showing differences between the IH and RA groups with $P$ values $\leq 0.08$ are shown in Table 1 .

\section{DISCUSSION}

The main findings of this study are that exposure to relatively mild IH during the early postnatal period in rat pups is associated with acute changes in systemic and brain parenchymal inflammatory cytokines and evidence for delayed medullary injury, DTI changes consistent with axonal injury and de- and/or dys-myelination, and MRS findings consistent with compromised cellular bioenergetics, neuronal integrity and transmission, and increased membrane turnover. To the best of our knowledge, this is the first report of a relationship between mild $\mathrm{IH}$, as might be encountered by a premature infant secondary to respiratory immaturity including apnea and periodic breathing, and adverse changes in systemic and brain inflammation, and brain structure and metabolism. 


\section{Inflammatory Response}

Compared with controls, IH-exposed pups manifested both a systemic and brain parenchymal proinflammatory response at P13 with increased plasma concentrations of CXCL1, and increased cerebellar IFN- $\lambda$ and IL-1 $\beta$. This acute response did not persist and was not evident by P18. CXCL1 is a

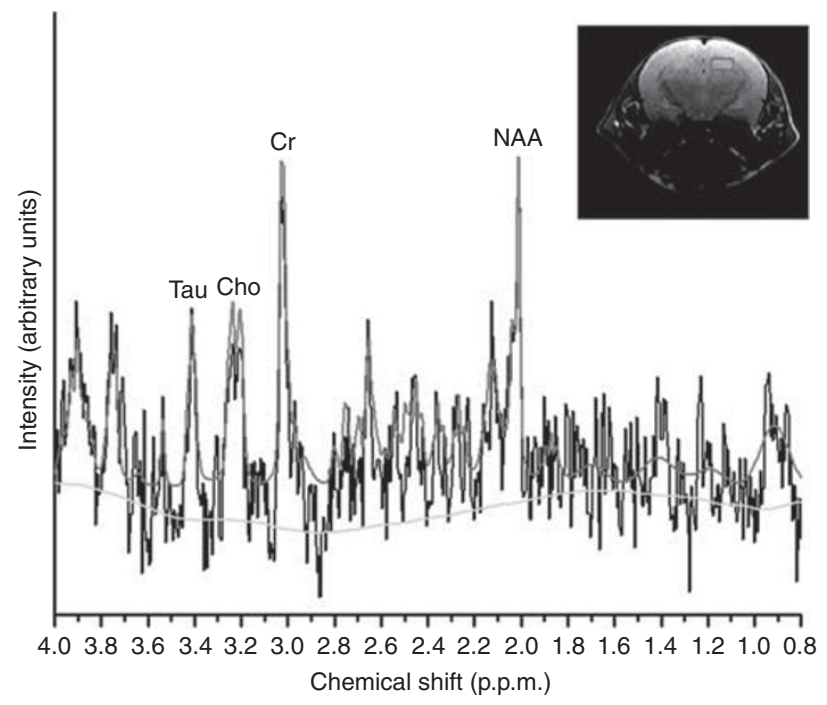

Figure 4. An example TARQUIN model spectrum from the hippocampus in a P22 control rat pup. The $x$ axis shows the chemical shift for each peak. Peaks for Creatine (Cr), Choline (Cho), Taurine (Tau), and $\mathrm{N}$-acetyl-aspartate (NAA) are shown. The inset shows the region of interest including the left hippocampus. chemokine that is a member of a family of small molecular mass proteins that regulate leukocyte migration and activation, and are stimulated by TNF- $\alpha$ (ref. 15). GRO- $\alpha$ is also a CXC neutrophil-specific $\alpha$-chemokine with an important role in inflammatory conditions (16). In the brain, CXCL1 plays a critical role in regulating proliferation and dispersal of oligodendrocyte progenitors, and in inhibiting oligodendrocyte migration during development (17). However, there was only a suggestion of an increase in CXCL1 in the cerebellum. It is unclear why there was not a more pronounced elevation in CXCL1 in the brain. It is possible that the signal was diluted because it is produced within small subregions of the larger regions we examined, or that the timing in the brain vs plasma is not entirely coincident. Additional temporal studies with subregional dissection are necessary to resolve this issue. Nevertheless, given the robust elevations in plasma CXCL1, we conclude that plasma elevations represent an acute systemic response to $\mathrm{IH}$. Overall, our results are consistent with acute inflammation and inflammation-associated brain injury.

\section{Brain Injury}

In the older p18 pups, NSE was higher in the medullas of IHexposed compared with RA controls. NSE is a glycolytic isoenzyme, with a high concentration in mature central and peripheral neurons. It is released after neuronal death (5) and is a known marker of ischemic brain damage in adults, specifically after traumatic brain injury, stroke, and anoxic encephalopathy after cardiac arrest $(18,19)$. The elevations in IFN- $\gamma$ and IL-1 $\beta$ that we observed have also been associated

Table 1. Metabolite concentration changes that were significant or had $P$ values of $\leqslant 0.08$ following exposure to intermittent hypoxia

\begin{tabular}{|c|c|c|c|c|c|c|c|c|}
\hline \multirow[t]{2}{*}{ Metabolite } & \multicolumn{4}{|c|}{ Brainstem } & \multicolumn{4}{|c|}{ Hippocampus } \\
\hline & RA & $\mathbb{H}$ & $P$ & & RA & $\mathbb{H}$ & $P$ & \\
\hline $\mathrm{Cr}$ & $3.94 \pm 0.54$ & $3.04 \pm 1.37$ & 0.030 & $\downarrow$ & $4.12 \pm 1.29$ & $4.00 \pm 1.72$ & 0.880 & \\
\hline $\mathrm{TCr}$ & $4.97 \pm 2.37$ & $7.16 \pm 1.04$ & 0.030 & $\uparrow$ & $8.67 \pm 1.76$ & $8.67 \pm 1.30$ & 1.00 & \\
\hline NAA/Cho & $8.90 \pm 3.18$ & $5.87 \pm 2.07$ & 0.042 & $\downarrow$ & $12.81 \pm 5.59$ & $7.62 \pm 2.83$ & 0.037 & $\downarrow$ \\
\hline TNAA/Cr & $3.99 \pm 2.61$ & $2.14 \pm 0.57$ & 0.060 & $\downarrow$ & $2.40 \pm 0.60$ & $1.88 \pm 0.42$ & 0.070 & \\
\hline NAA & $4.07 \pm 1.30$ & $5.20 \pm 0.91$ & 0.070 & $\uparrow$ & $5.90 \pm 1.87$ & $7.29 \pm 1.99$ & 0.190 & \\
\hline TCho & $0.84 \pm 0.28$ & $1.57 \pm 0.37$ & 0.001 & $\uparrow$ & $1.47 \pm 0.42$ & $1.12 \pm 0.25$ & 0.090 & \\
\hline pCho & $0.35 \pm 0.20$ & $0.59 \pm 0.24$ & 0.060 & $\uparrow$ & $0.64 \pm 0.33$ & $0.59 \pm 0.25$ & 0.740 & \\
\hline Gly/Cr & $2.64 \pm 2.10$ & $1.02 \pm 0.30$ & 0.040 & $\downarrow$ & $0.56 \pm 0.45$ & $0.79 \pm 0.38$ & 0.290 & \\
\hline GABA/Cr & $2.53 \pm 1.71$ & $1.28 \pm 0.69$ & 0.070 & $\downarrow$ & $1.37 \pm 0.65$ & $1.22 \pm 0.37$ & 0.580 & \\
\hline $\mathrm{Glc} / \mathrm{Cr}$ & $0.72 \pm 0.76$ & $0.20 \pm 0.25$ & 0.070 & $\downarrow$ & $0.20 \pm 0.24$ & $0.23 \pm 0.18$ & 0.760 & \\
\hline Ins/Cr & $3.47 \pm 2.87$ & $1.71 \pm 0.67$ & 0.080 & $\downarrow$ & $1.02 \pm 0.59$ & $0.92 \pm 0.33$ & 0.700 & \\
\hline
\end{tabular}

Cho, choline; Cr, creatine; Gly, glycine; GABA, $\lambda$-aminobutyric acid; GPC, glycerophosphocholine; Ins, inositol; NAA, N-acetyl-aspartate; p, phosphoryl; T, total; Tau, taurine. Arrows indicate direction of change. Significant values are given in bold. Values are $\mathrm{mM}$. 


\section{Articles | Darnall et al.}

with severe hypoxic-ischemia in human infants (20) and elevations of NSE, along with proinflammatory cytokines, are predictive of abnormal outcomes (21). However, our study examined the effects of more mild $\mathrm{IH}$ and the elevation in NSE was delayed compared to that of the proinflammatory markers. We had no measurements between P13 and P18, however, and the elevation in NSE may have occurred much earlier. Although elevations of NSE and inflammatory markers appear to occur concurrently in severe hypoxicischemic injury, it is possible that with exposure to more mild chronic IH, elevation in NSE is related more to the cumulative effect rather than to a relatively short severe exposure. We believe that our results, nevertheless, provide biochemical evidence of brain injury in our rodent model after a relatively mild exposure to $\mathrm{IH}$.

\section{Diffusion Tensor Imaging}

Although DTI has become a valuable tool to evaluate hypoxic injury in the human neonate (22), there are no DTI studies that have focused on mild $\mathrm{IH}$ associated with immature control of breathing, including periodic breathing. Our results are consistent with those of Juliano, et al (11) and Cai et al (12) in mice who found biochemical and electron microscopy evidence for impaired axonal myelination. Another study in rats, using DTI, studied the combination of hyperoxia and mild $\mathrm{IH}$, and reported transient adverse MRI changes in RD, $\mathrm{AD}$, and $\mathrm{FA}$ in certain white matter regions that did not persist beyond P14 (13). Unfortunately, there was no sustained hyperoxia control in this study, and therefore it was not possible to distinguish between the effects of sustained hyperoxia (23) and the added effects of mild intermittent hypoxia. We believe that our study is the first to examine in a neonatal rodent model the effects of mild $\mathrm{IH}$ on white matter integrity with DTI. In summary, we found overall decreases in $\mathrm{FA}$ and $\mathrm{AD}$ and increases in $\mathrm{RD}$ in selected white matter regions in our pups. The changes in $\mathrm{FA}, \mathrm{AD}$, and $\mathrm{RD}$ were significant in the CC and in FA and AD in the CG and are indicative of axonal pathology and de- or dys-myelination.

FA is a DTI measure frequently reported in human studies and is a summary measure of the microstructural integrity, but is not specific to the type of injury or change. AD tends to be variable in white matter changes and pathology, and usually decreases with axonal injury and increases with maturation. RD increases have been associated with white matter de- or dys-myelination. MD is an inverse measure of the membrane density and is sensitive to cellularity, edema, and necrosis (24). Thus, our findings suggest that exposure to levels of $\mathrm{IH}$, similar to what might be experienced during prematurity from 28 to 40 weeks PMA in a human infant, can result in axonal injury and de- or dys-myelination consistent with impairment of white matter integrity, which persist into later infancy.

\section{Magnetic Resonance Spectroscopy}

Exposure to mild intermittent hypoxia resulted in a decrease in the concentration of $\mathrm{Cr}$ and an increase in the $\mathrm{TCr}$ and
TCho (pCho+Cho) in the brainstem. Creatine and pCr play a key role in cellular energy homeostasis by replenishing ATP through the reversible transfer of a phosphoryl group from $\mathrm{pCr}$ to ADP upon creatine kinase activity (25). The observed changes in the $\mathrm{Cr}$ and $\mathrm{pCr}$ concentrations are consistent with their role in maintaining ATP levels required for the protection of brain tissue from hypoxic insult (14). Hypoxia also enhances the hydrolysis of Cho-containing phospholipids resulting in increased choline concentrations, and further Cho is phosphorylated to pCho (26). Increases in Cho-containing phospholipids along with a supporting but nonsignificant increase in pCho in the brainstem and changes in GPC in the brainstem and hippocampus suggest that exposure to $\mathrm{IH}$ increased membrane turnover. In other disease states, this has been associated with gliosis, demyelination, and inflammation (27). The significant decrease in NAA/Cho in both the brainstem $(P=0.042)$ and hippocampus $(P=0.037)$ are similar to findings in patients with OSA (28). Importantly, there is a significant correlation between brain NAA/Cho levels and the neurodevelopmental outcome in premature infants (29). In addition, there were important supporting but nonsignificant decreases in the ratios of NAA containing metabolites to $\mathrm{Cr}$ in both the brainstem and hippocampus. Taken together, these results support alterations in both neuronal and white matter integrity.

The decrease in Tau/Cr $(P=0.027)$ in the brainstem after exposure to IH may indicate an increased vulnerability to hypoxic injury. There were also significant increases in Gly in the hippocampus $(P=0.03)$ and decreases in $\mathrm{Gly} / \mathrm{Cr}$ in the brainstem $(P=0.04)$. Glycine is an important inhibitory neurotransmitter that also enables optimum NMDA receptor activity. Thus, exposure to IH may have important effects on neurotransmission.

There was a suggestion that NAA increased rather than decreased in the brainstem. Concentrations of NAA characteristically decrease in most disease states. Thus, our finding of a possible increase is unusual. It is possible that increases or decreases in any metabolite might occur over the time course of the same pathological process. One study in adult rats with repeated MRIs performed at 1,7 , and 14 days after exposure to $48 \mathrm{~h}$ of hypobaric hypoxia did not observe decreases in NAA and Cr until at least 7 days after exposure (30). The timing of these changes may well be different in our rodent pup model. Our data were obtained at a single time point $\sim 10$ days after completing the hypoxia exposure. However, there are little data in developing rodents using our experimental paradigm, and thus the reason for the disparity in the NAA findings remains unclear. Nevertheless, our findings do show relatively consistent evidence of hypoxiarelated alterations in cellular energetics, neuronal integrity, neurotransmission, and increased vulnerability to hypoxia.

There have been no investigations of MRS after mild IH in developing rodents patterned to mimic immature control of breathing with which to compare our findings. Other investigators, however, have reported changes in metabolites similar to our findings after more severe exposure to hypoxia 
in older animals, and in human patients with OSA. Douglas et al. (31) reported decreases in cerebral NAA/Cr ratio in 30day-old (P30) mice exposed to 4 weeks of IH using a protocol similar to that used in this study. There were no changes, however, after the exposure to chronic sustained hypoxia. On the other hand, other investigators have reported decreases in $\mathrm{Cr}$ and NAA at P21 in rat hippocampus after continuous exposure to $10 \%$ oxygen starting at P3 (ref. 32). In human patients with OSA, altered cognitive functions have been associated with structural-metabolic changes in certain regions of the brain (33). A recent meta-analysis of MRS findings in these patients showed consistent decreases in the $\mathrm{NAA} / \mathrm{Cr}$ ratio in the hippocampus (28). Consistent with our data, consistent decreases in NAA/Cho have also been reported in OSA patients $(27,28,34)$.

Most of the metabolite changes associated with $\mathrm{IH}$ occurred in the brainstem, although some changes did occur in the hippocampus. The hippocampus has been the usual focus of IH in adult patients with OSA (28) and appears, in the adult human, to be very sensitive to hypoxia. Interestingly, however, brainstem gliosis was described as early as in 1976 in many infants who died of the Sudden Infant Death Syndrome (SIDS) (35) and formed the basis for the hypothesis that intrauterine or postnatal hypoxia was involved in the etiology of SIDS. Although we did not examine our brainstems for gliosis, the metabolite findings that we observed are consistent with the idea that exposure to even mild IH can compromise brainstem function.

Although our findings are highly suggestive of proinflammatory changes and persisting impairment of white matter integrity and metabolic changes after exposure to mild IH in a rodent model, a number of issues require caution in their interpretation. In the living rodent, MRI must be performed under anesthesia. Inhalation anesthesia is used most commonly, as in this study, at a dose that allows spontaneous breathing. We used a low dose of isoflurane (2\%) in oxygen, which is in the range suggested by Flecknell (36). The small brain sizes in our animal make it difficult to be sure that we captured only the areas of interest. In addition, the quantification of metabolic information obtained from MRS is challenging because of both spectral and other issues, including the overlay of resonances, spin interaction, variable metabolite relaxation times, choice of line-fitting for numerical area-under-curve determination, and absolute quantification using external/internal standards (37). Resolution is one of the largest challenges of imaging small mammals, and the small voxel sizes greatly decrease the signal-to-noise ratio. This is partially overcome by using a magnet with a higher field power, such as the 9.4-tesla imaging system used in this study, which increases the signal-to-noise ratio by a factor of 5 compared with a 3-tesla scanner commonly used in human imaging. This is partly counterbalanced by a disadvantageous change in relaxation imaging parameters, and an increased tendency for disruptive artifact-generating factors. Nevertheless, the spectra that we generated (Figure 4) were comparable to those used as examples found in both human (29) and animal (38) literature.

In summary, our findings in rodent pups exposed to mild IH of a magnitude that might occur during apnea and periodic breathing during a developmental period, analogous to a premature infant between 28 and 40 weeks PMA, was associated with an increase in proinflammatory markers, DTI evidence for axonal injury and de- or dys-myelination, and MRS findings consistent with alterations in bioenergetics, decreases in neuronal integrity and transmission, increases in membrane turnover, and an increased vulnerability to hypoxic injury. Many premature infants born at $<34$ weeks gestation are exposed to similar levels of $\mathrm{IH}$ associated with apnea of prematurity and especially periodic breathing (3), which can persist beyond term equivalent age and is frequently not apparent $(2,39)$. Our data suggest that there may be significant deleterious effects of this persisting $\mathrm{IH}$, but future studies in human infants born preterm are needed to determine whether persisting IH due to immature breathing also causes acute injury and, more importantly, whether this acute injury is associated with long-term neurodevelopmental impairment.

\section{METHODS}

\section{Animals}

All procedures were approved by the Animal Care and Use Committee of Dartmouth College and conformed to ethical standards. Eight pregnant Sprague-Dawley rats (Harlan Laboratories) were obtained 3-4 days before delivery and housed under standard conditions in the Dartmouth College Animal Facility. After delivery, on postnatal day (P)2, after assuring that pups were nursing well, litters were culled to 10 pups. The dam and pups were then transferred to a satellite facility. In both facilities lighting was on between $7 \mathrm{AM}$ and $7 \mathrm{PM}$, room temperature was kept at $72 \pm 2{ }^{\circ} \mathrm{F}$, and food and water were provided ad libitum. For the examination of biomarkers of acute inflammation and injury, four dams and their litters were placed in chambers that provided exposure to either IH (2 litters; IH group) or to room air (2 litters; RA group) from P2 until P12-the approximate equivalent of term gestation in a human infant. After P12, the dams and litters were returned to the animal facility and given routine care. For the MRI studies an additional 4 dams and litters were studied: two from the IH group and two from the RA group. The exposures and environments were identical to those for the inflammatory marker studies. Both male and female rats were used for all studies.

\section{Experimental Protocols}

Intermittent hypoxia (IH). A total of eight dams and their litters were exposed to either IH or RA. For the exposure to IH, a computerized system (Biospherix, LTD. Parish, NY, USA) was used to provide a customized pattern that has been previously described (40) to closely approximate what is experienced by premature infants with apnea and periodic breathing (11). The system was programmed to provide 6 decreases in chamber oxygen concentration $\left(\mathrm{CHO}_{2}\right)$ per hour alternating with an hour of normoxia (Figure 5a). Each decrease in $\mathrm{CHO}_{2}$ lasted $\sim 3 \mathrm{~min}$, allowing $\sim 7 \mathrm{~min}$ of room air exposure between events. With each exposure oxyhemoglobin saturation (SAT) decreased below $90 \%$ for $\sim 2$ min and below $85 \%$ for $\sim 1.5 \mathrm{~min}$, and a nadir SAT of $\sim 55 \%$ (Figure $5 \mathbf{b}$ ). Thus, in each $24 \mathrm{~h}$ period, there were 72 hypoxia events resulting in a SAT below $85 \%$ for $108 \mathrm{~min}$, or $7.5 \%$ of the time. On average for each hour, SATS were below $90 \%$ for $5 \mathrm{~min}$ and below $85 \%$ for $4.5 \mathrm{~min}$. This level of hypoxia exposure compares favorably with the data reported by 


\section{Articles $\mid$ Darnall et al.}

Juliano, et al (11) for human premature infants born at 24-27 weeks gestation over the first 6 weeks of life. Exposure to RA was accomplished in identical chambers where air flow, noise, and pressure changes mimicked those during $\mathrm{IH}$ exposure, except that an intermittent influx of room air was used instead of nitrogen.

Inflammatory and brain injury biomarkers. Pups were studied either at $\mathrm{P} 13$, roughly equivalent to a term human infant, or at P18, roughly equivalent to early infancy. At each age, 4 pups from each of two litters from the IH group and two litters from the RA group (total of $N=8$ for each group) were removed from the litter and euthanized
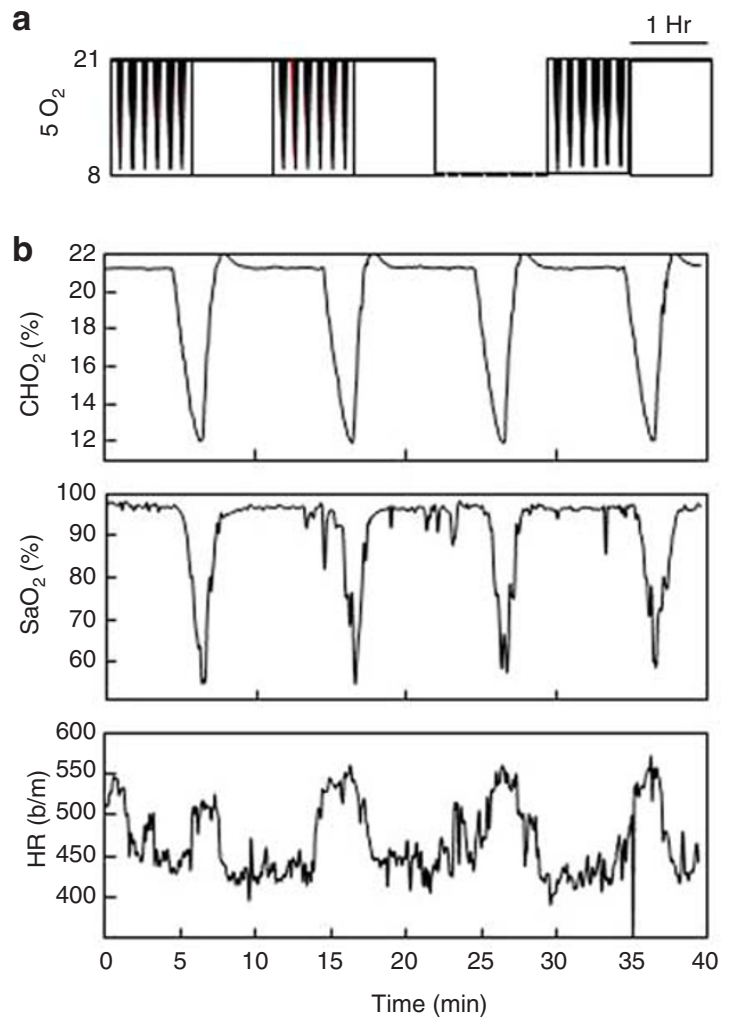

C

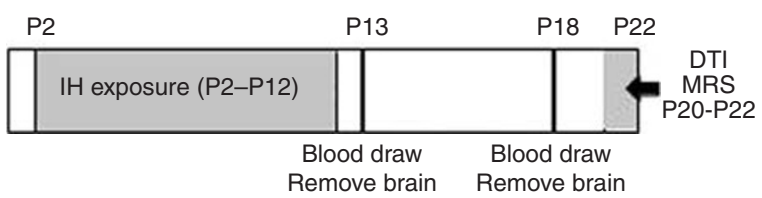

Figure 5. Pattern of intermittent hypoxia, resulting changes in chamber oxygen concentration, oxyhemoglobin saturation and heart rate, and study time line. (a) Pattern of intermittent hypoxia. There are 6 decreases to $\sim 6 \%$ per hour alternating with an hour of normoxia, $24 \mathrm{~h} /$ day, 7 days/week. Each decrease lasts $\sim 3$ min, resulting in 72 hypoxia events per day. (b) Decreases in chamber oxygen concentration $\left(\mathrm{CHO}_{2}\right)$, oxyhemoglobin saturation $\left(\mathrm{SaO}_{2}\right)$, and associated increases in heart rate (HR) with each decrease. (c) Timeline for the intermittent hypoxia (IH) exposure, biomarkers of acute injury, and DTI/MRS protocols. under pentobarbital anesthesia $(0.1 \mathrm{ml}-39 \mathrm{mg} / 100 \mathrm{mg}$ body weight, i.p.). EDTA blood was obtained by cardiac puncture; samples were centrifuged and the resultant plasma was divided into aliquots (50$100 \mu \mathrm{l})$ in tubes and immediately frozen $\left(-80{ }^{\circ} \mathrm{C}\right)$. Brains were removed and bisected into two halves. The left half of each brain was divided into cortex, cerebellum, and brainstem. Brain and blood samples were immediately frozen $\left(-80{ }^{\circ} \mathrm{C}\right)$ for later analysis (SK). Frozen brain regions were homogenized in RIPA buffer on ice with an added protease inhibitor cocktail (both from Sigma-Aldrich, St Louis, MO, USA). Samples were centrifuged at 17,200 $g$ for $15 \mathrm{~min}$ at $4{ }^{\circ} \mathrm{C}$, and the resulting supernatants were divided into aliquots $(15 \mu \mathrm{l})$ and frozen at $-80^{\circ} \mathrm{C}$. Proteins were later quantified with a Bradford protein assay (Bio-Rad, Hercules, CA, USA). Inflammatory cytokines were measured in duplicate in equivalent plasma volumes $(35 \mu \mathrm{l})$ or $120 \mathrm{mg}$ protein aliquots from brain tissue, using a V-Plex Plus Proinflammatory Rat Multiplex ELISA (MesoScale Diagnostics, Rockville, MD, USA) measuring IFN- $\gamma$, IL- $1 \beta$, IL-4, IL-5, IL-6, IL-10, IL-13, KC/GRO, and TNF- $\alpha$. As an indicator of brain injury, neuronspecific enolase (NSE) was measured in equivalents of $100 \mu \mathrm{g}$ of protein, using an ELISA (Cloud-Clone, Atlanta, GA, USA). The timeline for the inflammatory marker and brain imaging protocols is shown in Figure 5c.

\section{Brain Imaging}

MRI parameters. To assess possible persisting alterations in white matter structure and brain metabolism, studies were performed at P20-P22 using a 9.4T Agilent small animal MRI system equipped with triple axes gradients (Agilent, Palo Alto, CA, USA) and a 4-channel rat head phased-array coil (Rapid Biomedical $\mathrm{GmbH}$, Würzburg, Germany). Rat pups were anesthetized with isoflurane inhalation ( $2 \%$ in oxygen) and the body temperature was maintained at $37^{\circ} \mathrm{C}$ using a small rodent heater system (SAII, Stony Brook, NY).

Diffusion tensor imaging (DTI). A total of nine pups were randomly chosen from 2 litters in the IH group and 11 pups were chosen from 2 litters in the RA group for study. In addition to one $b=0$ scan, we used 6 gradient directions with $b=1000 \mathrm{~s} / \mathrm{mm}^{2}$ and a slice thickness of $1 \mathrm{~mm}$ and an in-plane resolution of $\sim 160 \times 160 \mu \mathrm{m}$. For each voxel in each central white matter region of interest (Figure 2a), a three-dimensional tensor in the form of an ellipsoid was derived. The axes of the ellipsoid are described by a set of eigenvectors and eigenvalues. In white matter, tensors are elongated (anisotropic) and aligned with fiber tracts, whereas in gray matter, tensors tend to be more spherical. Values for $\mathrm{RD}, \mathrm{AD}$, mean diffusivity (MD), and FA were derived from the corresponding eigenvalues $\left(\lambda_{\mathrm{i}}\right)$ and averaged for each region of interest.

Magnetic resonance spectroscopy. From additional litters at P20P22 brain metabolite profiles were acquired from nine pups in the IH group and six pups in the RA group. Two sets of spectra, with and without water suppression, were acquired from the brainstem and hippocampus for each animal. We chose to focus on the brainstem because of our interest in respiratory control in premature infants and on the hippocampus because of the known effects of IH in hippocampus in adult OSA patients. The water-suppressed MRS parameters were as follows: $\mathrm{TR}=2,000 \mathrm{~ms}, \mathrm{TE}=14 \mathrm{~ms}$, spectral width $=4006 \mathrm{~Hz}$, number of complex points $=1,024$, voxel size $=3.6$ $\mathrm{mm} \times 1.4 \mathrm{~mm} \times 3.2 \mathrm{~mm}$, and number of averages $=128$. Water suppression was performed using the variable power and optimized relaxation delays (VAPOR) scheme. The corresponding reference

Table 2. Metabolites, lipids, and macromolecules quantified using TARQUIN

Alanine, Aspartate, Citrate, Creatine ( $\mathrm{Cr}$ ), $\gamma$-aminobutyric acid (GABA), Glucose (GIc), Glutamine, Glutamate, Glutathione, Glycerophosphocholine (GPC), Glycine (Gly), m-Inositol, Lactate, $\mathrm{N}$-acetyl-aspartate (NAA), N-acetylaspartyl glutamate (NAAG), Phosphoethanolamine (Choline, Cho), Phosphocholine (pCho), Phosphocreatine (pCr), Scyllo-Inositol, Taurine (Tau)
Lipid 09, Lipid 13a, Lipid 13b, Lipid 20 Macromolecule 09, Macromolecule 12, Macromolecule 14, Macromolecule 17, Macromolecule 20, Macromolecule 38 
spectra without water suppression were recorded using identical parameters.

Metabolites were quantified from spectra using TARQUIN (Totally Automatic Robust Quantitation in NMR) version 4.3.8 with a basis set including 20 metabolites and 10 lipid and macromolecular components (Table 2). The amplitudes of the metabolite signals were estimated in absolute units ( $\mathrm{mM})$, by scaling the fitted signal amplitudes to the amplitude of an unsuppressed water resonance. The ratios of metabolites to creatine $(\mathrm{Cr})$ were also compared between the two groups. The ratio of $\mathrm{N}$-acetyl-aspartate to choline (NAA/Cho) was also compared because of reports of significant decreases in NAA/Cho in human sleep apnea cases (33) and the significant correlation of NAA/Cho with later mental development in infants born preterm (29).

\section{Statistics}

Comparisons were made with $T$-tests with appropriate corrections for multiple comparisons. We used an alpha of $P<0.05$ to assess significance but also reported interesting but nonsignificant differences for completeness and to identify consistent trends. We arbitrarily included values with a chance of $\leq 8$ out of a 100 that the observed observations would reject the null hypothesis $(P \leq 0.08)$.

\section{ACKNOWLEDGMENTS}

We thank Luxi Xia for helping us with the animals. The data have been partially presented at the Experimental Biology Annual meeting in April 2016, the PAS meeting in May 2016, and the MRS results at the SPIE. Medical Imaging meeting in February, 2017 (Darnall RA et al, Proc SPIE Int Soc Opt Eng 10137:101371Y, 2017).

\section{STATEMENT OF FINANCIAL SUPPORT}

These studies were supported by grants from the NIH, NIH PO1 HD36379; The American SIDS Institute; and The Hearst Foundation, Department of Pediatrics, Dartmouth Geisel School of Medicine, NIH NCMRR/NINDS 2R24HD050846-06 (NCMRR-DC Core Molecular and Functional Outcome Measures in Rehabilitation Medicine). 9.4T Magnet for MRI studies was funded by NIH 1S10RR025048-01.

Disclosure: The authors declare no conflict of interest

\section{REFERENCES}

1. Poets CF, Roberts RS, Schmidt B, et al. Association between intermittent hypoxemia or bradycardia and late death or disability in extremely preterm infants. JAMA 2015;314:595-603.

2. Hunt CE, Corwin MJ, Weese-Mayer DE, et al. Longitudinal assessment of hemoglobin oxygen saturation in preterm and term infants in the first six months of life. J Pediatr 2011;159:377-83 e371.

3. Patel M, Mohr M, Lake D, et al. Clinical associations with immature breathing in preterm infants: part 2-periodic breathing. Pediatr Res 2016;80:28-34.

4. Nagata N, Saji M, Ito T, Ikeno S, Takahashi H, Terakawa N. Repetitive intermittent hypoxia-ischemia and brain damage in neonatal rats. Brain Dev 2000;22:315-20.

5. Douglas-Escobar M, Weiss MD. Biomarkers of brain injury in the premature infant. Front Neurol 2012;3:185.

6. Leviton A, Allred EN, Dammann O, et al. Systemic inflammation, intraventricular hemorrhage, and white matter injury. J Child Neurol 2013;28:1637-45.

7. Hunt CE. Neurocognitive outcomes in sleep-disordered breathing. J Pediatr 2004;145:430-2.

8. Neubauer JA. Invited review: physiological and pathophysiological responses to intermittent hypoxia. J Appl Physiol 2001;90:1593-9.
9. Julien CA, Joseph V, Bairam A. Alteration of carotid body chemoreflexes after neonatal intermittent hypoxia and caffeine treatment in rat pups. Respir Physiol Neurobiol 2011;177:301-12.

10. Chu A, Gozal D, Cortese R, Wang Y. Cardiovascular dysfunction in adult mice following postnatal intermittent hypoxia. Pediatr Res 2015;77: 425-33.

11. Juliano C, Sosunov S, Niatsetskaya Z, et al. Mild intermittent hypoxemia in neonatal mice causes permanent neurofunctional deficit and white matter hypomyelination. Exp Neurol 2015;264:33-42.

12. Cai J, Tuong CM, Zhang Y, et al. Mouse intermittent hypoxia mimicking apnoea of prematurity: effects on myelinogenesis and axonal maturation. J Pathol 2012;226:495-508.

13. Morken TS, Nyman AK, Sandvig I, et al. Brain development after neonatal intermittent hyperoxia-hypoxia in the rat studied by longitudinal MRI and immunohistochemistry. PLoS One 2013;8:e84109.

14. Wilken B, Ramirez JM, Probst I, Richter DW, Hanefeld F. Creatine protects the central respiratory network of mammals under anoxic conditions. Pediatr Res 1998;43:8-14.

15. Fox SE, Lu W, Masheshwari A, Christensen RD, Calhoun DA. The effects and comparative differences of neutrophil specific chemokines on neutrophil chemotaxis of the neonate. Cytokine 2005;29:135-40.

16. Sullivan SE, Staba SL, Gersting JA, et al. Circulating concentrations of chemokines in cord blood, neonates, and adults. Pediatr Res 2002;51: 653-7.

17. Omari KM, Lutz SE, Santambrogio L, Lira SA, Raine CS. Neuroprotection and remyelination after autoimmune demyelination in mice that inducibly overexpress CXCL1. Am J Pathol 2009;174:164-76.

18. Pleines UE, Morganti-Kossmann MC, Rancan M, Joller H, Trentz O, Kossmann T. S-100 beta reflects the extent of injury and outcome, whereas neuronal specific enolase is a better indicator of neuroinflammation in patients with severe traumatic brain injury. J Neurotrauma 2001;18:491-8.

19. Anand N, Stead LG. Neuron-specific enolase as a marker for acute ischemic stroke: a systematic review. Cerebrovasc Dis 2005;20:213-9.

20. Chalak LF, Sanchez PJ, Adams-Huet B, Laptook AR, Heyne RJ, Rosenfeld CR. Biomarkers for severity of neonatal hypoxic-ischemic encephalopathy and outcomes in newborns receiving hypothermia therapy. J Pediatr 2014;164:468-74, e461.

21. Ramaswamy V, Horton J, Vandermeer B, Buscemi N, Miller S, Yager J. Systematic review of biomarkers of brain injury in term neonatal encephalopathy. Pediatr Neurol 2009;40:215-6.

22. Massaro AN, Evangelou I, Fatemi A, et al. White matter tract integrity and developmental outcome in newborn infants with hypoxic-ischemic encephalopathy treated with hypothermia. Dev Med Child Neurol 2015;57:441-8.

23. Brehmer F, Bendix I, Prager S, et al. Interaction of inflammation and hyperoxia in a rat model of neonatal white matter damage. PLoS ONE 2012;7:e49023.

24. Feldman HM, Yeatman JD, Lee ES, Barde LH, Gaman-Bean S. Diffusion tensor imaging: a review for pediatric researchers and clinicians. J Dev Behav Pediatr 2010;31:346-56.

25. Koob M, Viola A, Le Fur Y, et al. Creatine, glutamine plus glutamate, and macromolecules are decreased in the central white matter of premature neonates around term. PLoS ONE 2016;11:e0160990.

26. Beley A, Bertrand N, Beley P. Cerebral ischemia: changes in brain choline, acetylcholine, and other monoamines as related to energy metabolism. Neurochem Res 1991;16:555-61.

27. Alkan A, Sharifov R, Akkoyunlu ME, et al. MR spectroscopy features of brain in patients with mild and severe obstructive sleep apnea syndrome. Clin Imaging 2013;37:989-2.

28. Xia Y, Fu Y, Xu H, Guan J, Yi H, Yin S. Changes in cerebral metabolites in obstructive sleep apnea: a systemic review and meta-analysis. Sci Rep 2016;6:28712.

29. Bapat R, Narayana PA, Zhou Y, Parikh NA. Magnetic resonance spectroscopy at term-equivalent age in extremely preterm infants: association with cognitive and language development. Pediatr Neurol 2014;51:53-9. 


\section{Articles $\mid$ Darnall et al.}

30. Koundal S, Gandhi S, Kaur T, Khushu S. Neurometabolic and structural alterations in rat brain due to acute hypobaric hypoxia: in vivo $1 \mathrm{H}$ MRS at 7T. NMR Biomed 2014;27:341-7.

31. Douglas RM, Miyasaka N, Takahashi K, Latuszek-Barrantes A, Haddad GG, Hetherington HP. Chronic intermittent but not constant hypoxia decreases NAA/Cr ratios in neonatal mouse hippocampus and thalamus. Am J Physiol Regul Integr Comp Physiol 2007;292:R1254-9.

32. Raman L, Tkac I, Ennis K, Georgieff MK, Gruetter R, Rao R. In vivo effect of chronic hypoxia on the neurochemical profile of the developing rat hippocampus. Brain Res Dev Brain Res 2005;156:202-9.

33. Bartlett DJ, Rae C, Thompson CH, et al. Hippocampal area metabolites relate to severity and cognitive function in obstructive sleep apnea. Sleep Med 2004;5:593-6.

34. Kamba M, Inoue Y, Higami S, Suto Y, Ogawa T, Chen W. Cerebral metabolic impairment in patients with obstructive sleep apnoea: an independent association of obstructive sleep apnoea with white matter change. J Neurol Neurosurg Psychiatry 2001;71:334-9.
35. Kinney HC, Burger PC, Harrell Jr FE, Hudson Jr RP. 'Reactive gliosis' in the medulla oblongata of victims of the sudden infant death syndrome. Pediatrics 1983;72:181-7.

36. Flecknell PA. Laboratory Animal Anaesthesia. Academic Press, 2016.

37. Huppi PS, Lazeyras F. Proton magnetic resonance spectroscopy $((1) \mathrm{H}-$ MRS) in neonatal brain injury. Pediatr Res 2001;49:317-20.

38. Hoyer C, Gass N, Weber-Fahr W, Sartorius A. Advantages and challenges of small animal magnetic resonance imaging as a translational tool. Neuropsychobiology 2014;69:187-201.

39. Rhein LM, Dobson NR, Darnall RA, et al. Effects of caffeine on intermittent hypoxia in infants born prematurely: a randomized clinical trial. JAMA Pediatr 2014;168:250-7.

40. Darnall RA, Schneider RW, Tobia CM, Zemel BM. Arousal from sleep in response to intermittent hypoxia in rat pups is modulated by medullary raphe GABAergic mechanisms. Am J Physiol Regul Integr Comp Physiol 2012;302:R551-60 